\title{
1 A scalable and continuous access to pure cyclic polymers enabled by 2 "quarantined" heterogeneous catalysts
}

3 Ki-Young Yoon ${ }^{1,4}$, Jinkyung Noh ${ }^{2,4}$, Quan Gan ${ }^{1,4}$, Julian P. Edwards ${ }^{1}$, Robert Tuba ${ }^{3}$, Tae-Lim Choi*,2, and 4 Robert H. Grubbs*,1

$5{ }^{1}$ Arnold and Mabel Beckman Laboratory of Chemical Synthesis, Division of Chemistry and Chemical

6 Engineering, California Institute of Technology, Pasadena, California 91125, the United States

$7 \quad{ }^{2}$ Department of Chemistry, Seoul National University, Seoul 08826, the Republic of Korea

$8{ }^{3}$ Institute of Materials and Environmental Chemistry, Research Centre for Natural Sciences, Magyar

9 tudósok körútja 2, P.O. Box 286, 1519 Budapest, Hungary.

$10 \quad{ }^{4}$ These authors contributed equally.

Cyclic polymers are topologically interesting and envisioned as a lubricant material.

13 However, scalable synthesis of pure cyclic polymers remains elusive. The most straightforward

14 way is to recycle a used catalyst for the synthesis of cyclic polymers. Unfortunately, it is

15 demanding because of the catalyst's vulnerability and inseparability from polymers, which

16 depreciates the practicality of the process. Here, we develop a continuous process streamlined in

17 a circular way that polymerization, polymer separation, and catalyst recovery happen in situ, to

18 dispense a pure cyclic polymer after bulk ring-expansion metathesis polymerization of

19 cyclopentene. It is enabled by introducing silica-supported ruthenium catalysts and a newly-

20 designed glassware. Also, different depolymerization kinetics of the cyclic polymer from its linear

21 analogue is discussed. This process minimizes manual labor, maximizes security of vulnerable 
catalysts, and guarantees purity of cyclic polymers, thereby showcasing a prototype of a scalable

access to cyclic polymers with increased reusability of precious catalysts ( $\geq 415,000$ turnovers).

The economy of lubrication is encumbered by a high replacement cost of lubricants in many applications ${ }^{1,2}$. One of its countermeasures is to increase the lifespan of lubricants, thereby decreasing the replacement frequency. The most common synthetic lubricant by far is polyalphaolefin ${ }^{3-5}$, which gradually loses its viscosity due to permanent chain scissions over time ${ }^{6}$. Cyclic hydrocarbon polymers similar to polyalphaolefins or mineral oils (e.g. polyethylene, polypropylene, polybutadiene, etc. $)^{7,8}$ are tribologically interesting because the initial chain scission of cyclic topology increases its viscosity by producing an opened linear topology with a higher chain volume ${ }^{9-11}$. This feature of cyclic polymers is envisioned as a viscosity modifier to prolong the lubricant lifetime. Since our discovery of the ring expansion route to cyclic polymers in $2002^{11}$, we $\mathrm{e}^{12-16}$ and other groups ${ }^{17-26}$ have done exciting research on more functionalized and purer cyclic polymers.

One of the most important needs is the development of a practical synthetic process to produce pure cyclic polymers on a larger scale for testing in many applications. To date, all the reported synthetic protocols were operated on a milligram scale in solution by homogeneous catalysis, which was accompanied by rigorous ex situ processes for polymer purification without actual catalyst recovery (Fig. 1a) ${ }^{24-27}$. Nonetheless, the residual metal catalysts remained at a few hundred ppm level ${ }^{12}$; in this conventional process, it is difficult not only to obtain an uncontaminated white polymer but also to recycle the precious metal catalysts. 
a
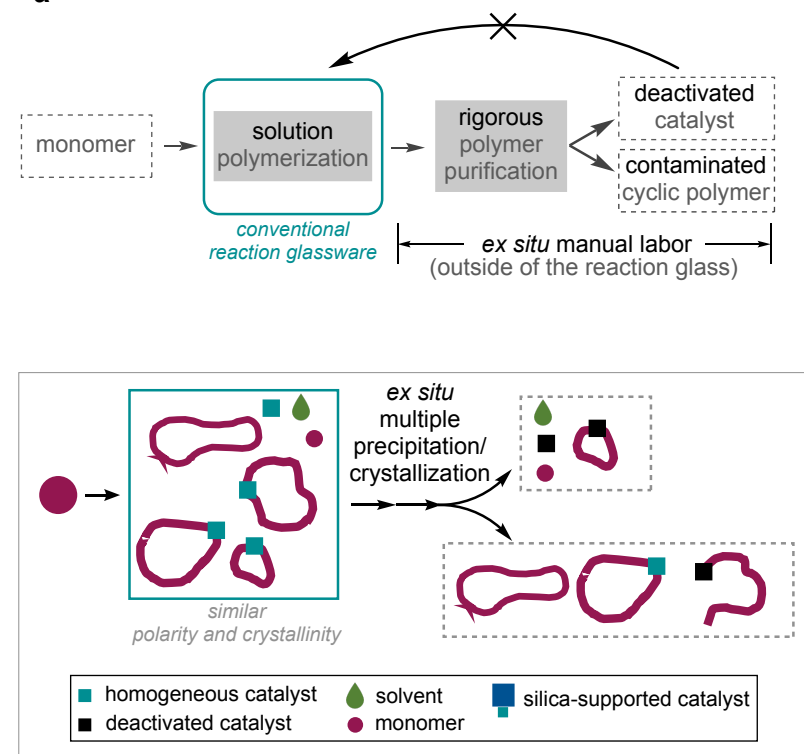

C

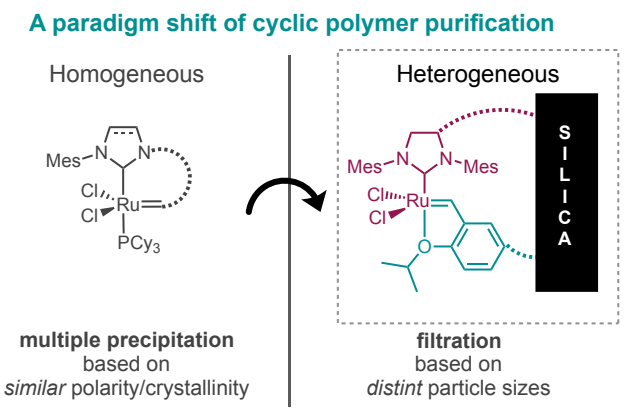
for REMP of cyclopentene in this work.
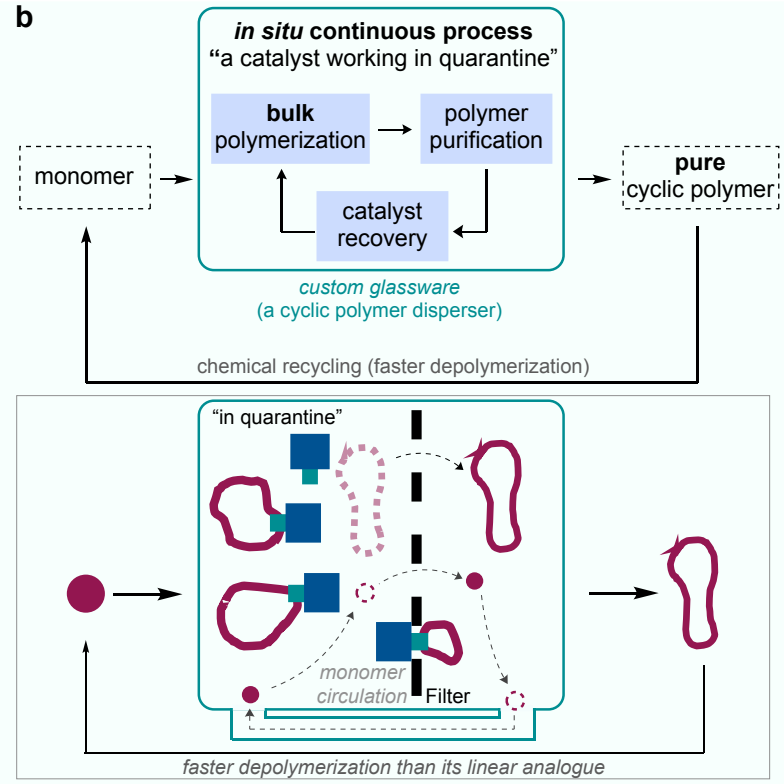

d

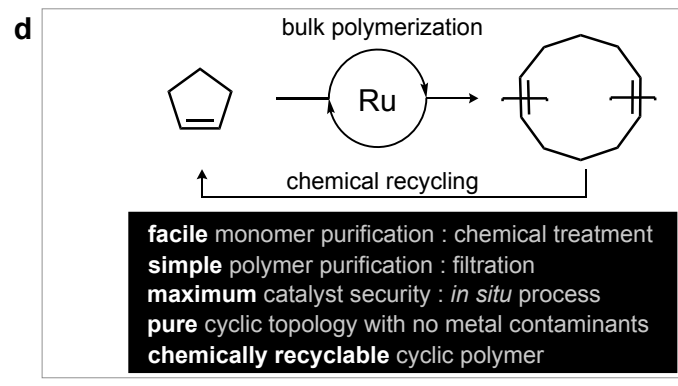

Fig. 1 REMP and purification process for the preparation of cyclic polymers. a, Conventional ex situ purification processes. b, In situ continuous process in this work. c, Catalyst design strategy. d, Key points

Here, we present a continuous synthetic process which is streamlined in a way that polymerization, polymer separation, and catalyst recovery simultaneously happen in situ in a closed loop (Fig. 1b), to dispense a cyclic polymer synthesized by bulk ring-expansion metathesis polymerization (REMP) of cyclopentene (CP) (Fig. 1d). To avoid extensive purification, silicasupported either cyclic or non-cyclic ruthenium catalysts are designed so that pure cyclic polymers can be segregated simply by filtration (Fig. 1C). Glassware that benefits most from the heterogeneous catalysts, namely a cyclic polymer dispenser, is customized to allow bulk 
1 polymerization with a concomitant separation of cyclic polymers from the heterogeneous

2 catalysts. A thimble containing the catalysts that is mounted as a cartridge on the dispenser is

3 situated in the original place throughout the process. This in situ continuous process minimizes

4 manual labor, quarantines the vulnerable catalytic species secured from any deactivating events

5 that can occur otherwise upon ex situ purifications, and guarantees purity of cyclic polymers.

6 Serendipitously, it is also found that the resulting cyclic polymer depolymerizes noticeably faster

7 than its linear analogue. Overall, this report demonstrates a prototype of a scalable and practical

8 access to a chemically-recyclable cyclic polymer with enlarged reusability of precious catalysts.

10 Results and Discussion

11 REMP catalyst design for simple polymer separation: from homogeneity to heterogeneity.

12 Previous REMP studies have adhered to a typical purification method conducted after ring13 opening metathesis polymerization (ROMP), which is multiple polymer precipitation or 14 crystallization in cold polar protic solvents ${ }^{24-27}$. The rigorous purification was inevitable because 15 polarity and crystallinity of cyclic polymers were not sufficiently distinct from undesired 16 polymeric metal complexes (e.g. $\mathbf{P 1}$ vs $\mathbf{H 1}$ in Fig. $2 \mathrm{~b}$ ). While this purification is still suitable for the

17 removal of metal which is already deactivated with a quencher in ROMP, it is in fact inappropriate

18 from the aspect of catalyst recovery in REMP in which the regenerated catalyst is active upon the

19 release of cyclic polymers. Furthermore, all the polymeric ruthenium species generated via the 20 side pathways are de facto active catalysts for REMP (Fig. 2b). Thus, the demanding post-

21 synthetic purification likely decomposes the metal catalysts by oxygen, moisture, or purification

22 solvents ${ }^{28}$, potentially followed by release of unwanted linear polymers or polymeric metal 
1a).
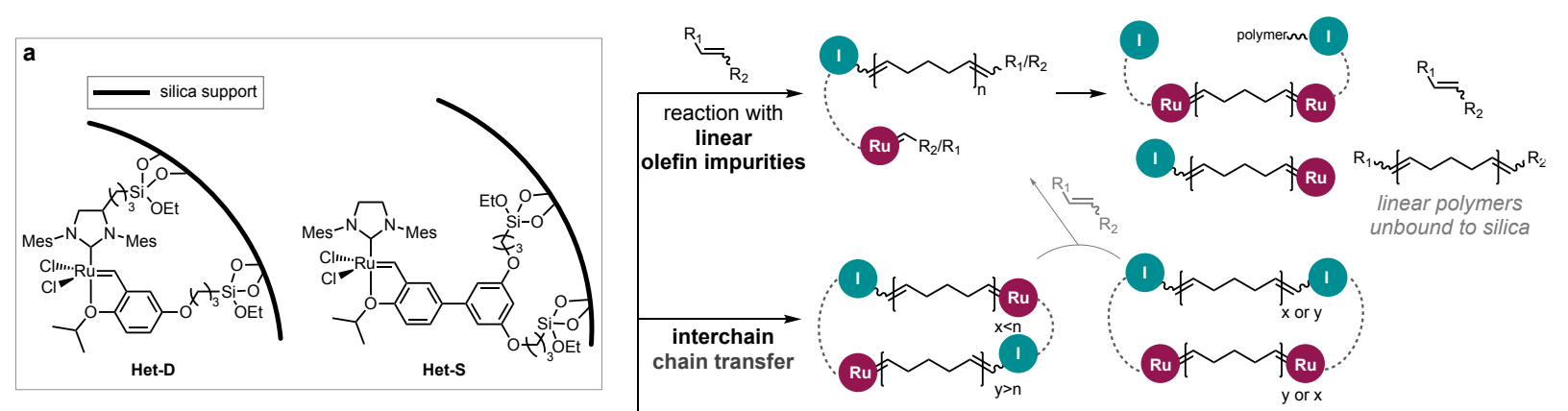

b
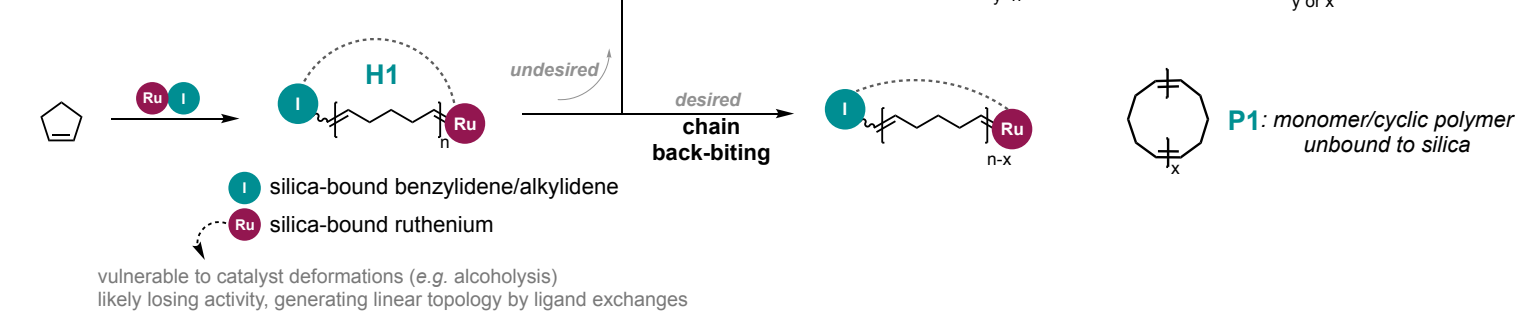

vulnerable to catalyst deformations (e.g. alcoholysis)
likely losing activity, generating linear topology by ligand exchanges

Fig. 2 REMP of CP with the immobilized ruthenium catalysts. a, The silica-supported ruthenium catalysts used in this study. $\mathbf{b}$, Possible olefin metathesis pathways of cyclopentene ring opening.

Previous ruthenium-based catalysts for REMP shared a common structure of metallacycle where the $\mathrm{N}$-heterocyclic carbene (NHC) and alkylidenes ${ }^{11-13} /$ benzylidenes $^{17}$ were tethered by organic linkers in the cyclic form (Fig. 1c). By replacing the organic linker with the silica gel support, we envisioned a simpler and more guaranteed separation of the desired cyclic polymer from the rest by filtration - the cyclic polymer is the only product chemically unbound from the silica support unless linear chain-transfer agents are present in the system (Fig. 2b). The envisioned catalyst, Het-D (a silica-containing metallacycle), was synthesized with the longest linear steps of ten (Fig. 2a, See Supplementary Information for the synthetic details). In addition, acyclic Het-S was prepared in fewer steps for comparison and found as effective (vide infra). These REMP catalysts consist of the chelating benzylidene structure, which are more stable in the air than the previous REMP catalysts. The amount of the ruthenium catalyst anchored onto silica support was 
1 determined from the Ru to Si ratio $(4.0 \mu \mathrm{mol}$ Ru/g silica for Het-D, $2.8 \mu \mathrm{mol}$ Ru/g silica for Het-S)

2 obtained by the inductively coupled plasma mass spectrometry (ICP-MS) (Table S1). The degree

3 of heterogeneity of the catalysts was confirmed with the hot filtration test ${ }^{29,30}$ (Table S2).

4

5 Monomer selection and purification: "garbage in, garbage out". Although known as a low-strain

6 monomer that is challenging for exergonic ROMP/REMP ${ }^{31-33}$, cyclopentene (CP) was selected as

7 the monomer for initial studies. Given a typical molar mass range of polyalphaolefins used for

8 lubrication $(<50 \mathrm{kDa})^{3}$, it is unnecessary to design higher-molar-mass cyclic polyCP/polyethylene

9 for viscosity modifier purposes. In this respect, limited monomer conversion, even in bulk

10 polymerization (e.g. $84 \%$ at r.t. ${ }^{31}$ ), which was considered a problem in previous studies aiming to

11 synthesize linear polyCP with higher molar masses $(>100 \mathrm{kDa})^{32,34,35}$, is no longer a drawback since

12 the low boiling-point cyclopentene can play the dual role of monomer and solvent with no need

13 of additional solvent. Furthermore, residual CP can be easily separated from polyCP by simple

14 distillation or evaporation under ambient conditions $\left(\mathrm{bp}=45^{\circ} \mathrm{C}\right)$. Moreover, the resulting polyCP

15 itself is interesting, as it is a chemically recyclable natural rubber analog, as well as a pre-

16 hydrogenated polymer of unique polyethylene featuring odd-numbered $\mathrm{CH}_{2}$ periodicity, which is

17 otherwise hard to access ${ }^{32}$.

Unfortunately, commercially available CP contained detectable amounts of acyclic olefin

19 impurities ( $2000 \mathrm{ppm})^{35}$. These impurities acting as chain transfer agents can not only decrease

20 the molar mass of polyCP ${ }^{35-37}$ but also deteriorate the purity of cyclic topology in the REMP

$21 \operatorname{process}^{16}$ (Fig. 3b). Previously, fractional distillation equipped with 28-inch Hempel column and

22 ceramic Berl saddles was exploited to separate these impurities from CP because of the proximal 
1 boiling points between them ${ }^{35}$. We envisaged that this strict distillation method could be

2 replaced with a simpler method, provided that a chemical treatment distinguishing acyclic olefins

3 from $\mathrm{CP}$ would be found. We selected the cobalt-catalyzed olefin isomerization-selective

4 hydroboration of terminal olefins developed by Chirik's group ${ }^{38}$; with only 0.001 mol\% of the

5 cobalt catalyst based on $\mathrm{CP}$, the acyclic olefin impurities were transformed into much heavier

6 hydroborated products via isomerized terminal olefins. On the other hand, CP remained intact

7 because the isomerization of CP never generates a terminal olefin (Fig. 3a) so that light CP could

8 be separated by simple distillation (Fig. 3b and S1).

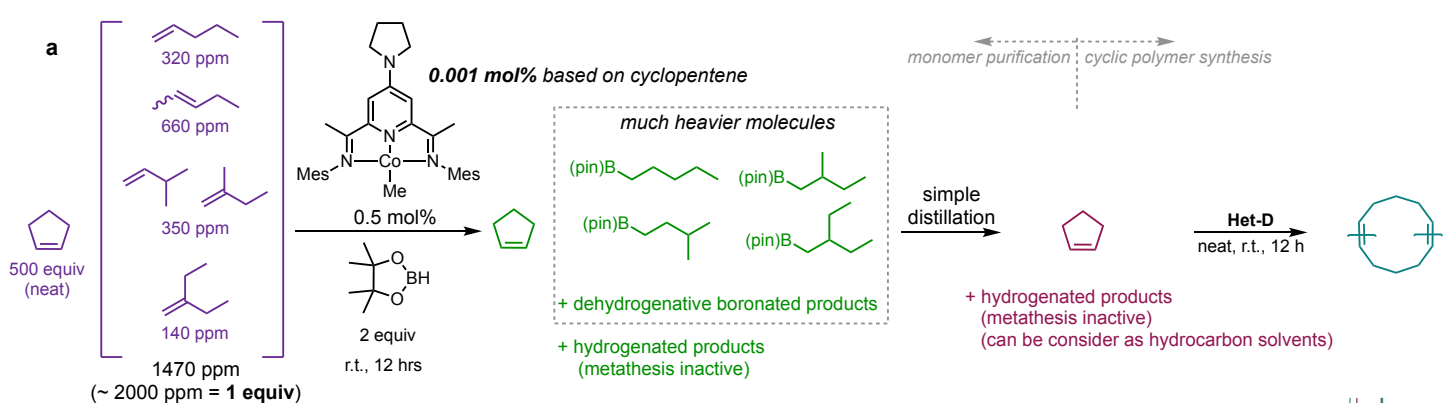

b

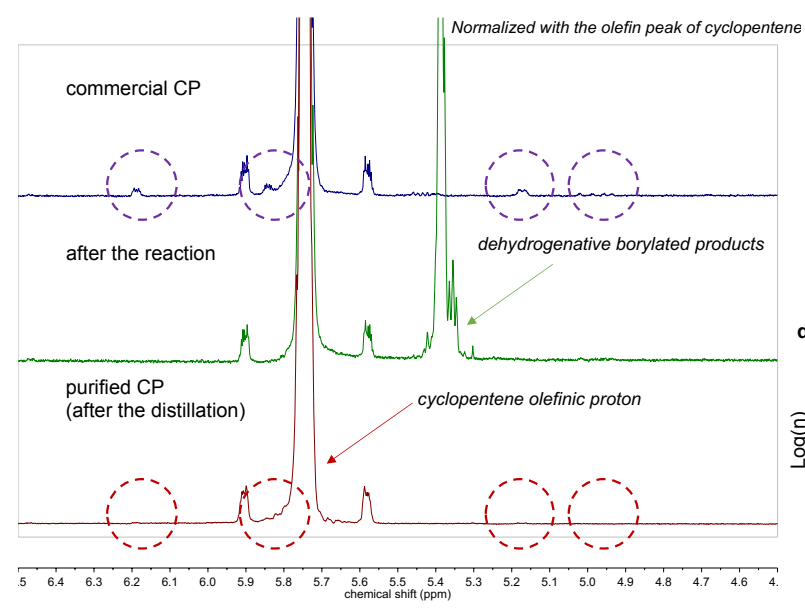

c

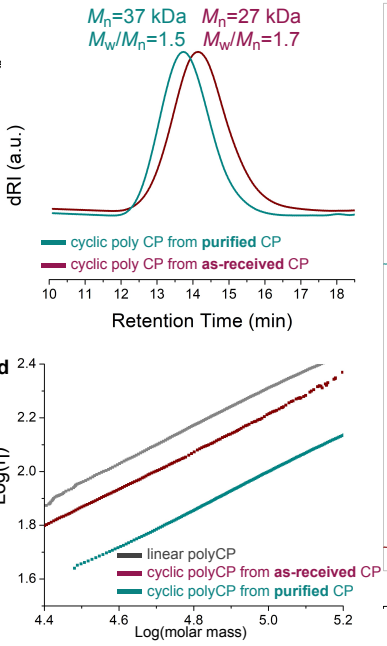

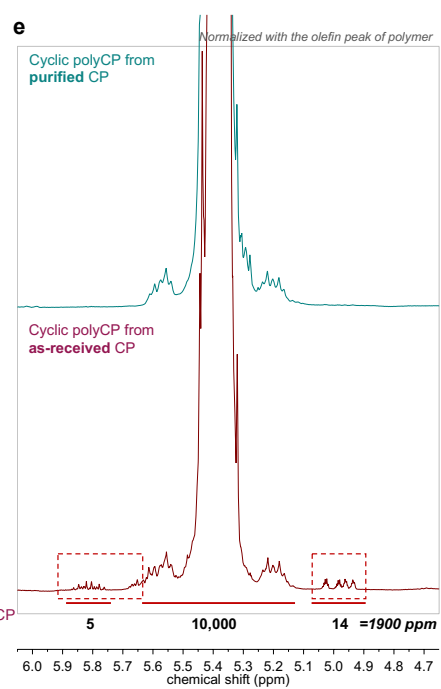

10 Fig. 3. Importance of monomer purity for REMP. a, A chemical treatment to differentiate CP from linear

11 olefin impurities. b, ${ }^{1} \mathrm{H}$ NMR spectra of olefinic regions before and after the hydroboration, as well as after

12 simple distillation. comparison of cyclic polyCP made of the purified CP $\left(M_{\mathrm{n}}=37 \mathrm{kDa}, M_{\mathrm{w}} / M_{\mathrm{n}}=1.5\right)$ vs cyclic

13 polyCP made of commercial CP as received $\left(M_{\mathrm{n}}=27 \mathrm{kDa}, M_{\mathrm{w}} / M_{\mathrm{n}}=1.5\right)$. c, SEC traces. d, Mark-HouwinkSakurada plots $\mathbf{e},{ }^{1} \mathrm{H}$ NMR spectra. 

and $20 \mathrm{mg}$ of Het-D $(0.08 \mu \mathrm{mol})$ at room temperature in a $20-\mathrm{mL}$ vial under an argon atmosphere. After filtration, the molar mass of the polymer made of commercial CP was found to be lower than that of the polymer from purified CP (Fig. 3c, see Fig. S2 for the similar result of the corresponding ROMP). Also, the cyclic topology was clearly deteriorated when commercial CP was used. The intrinsic viscosities $(\eta)$ of the two polyCPs over a range of molar masses, measured by size-exclusion chromatography (SEC) equipped with a differential viscometer, showed that the cyclic polyCP from purified CP $\left(\langle\eta\rangle_{\text {cyclic }} /\langle\eta\rangle_{\text {linear }}=0.44\right)$ had a lower intrinsic viscosity than the one from commercial CP over the whole range $\left(\langle\eta\rangle_{\text {cyclic }} /\langle\eta\rangle_{\text {linear }}=0.81\right)$, which indicated linear contaminants in the polymer from commercial CP (Fig. 3e, S3 and Table S3). The ${ }^{1} \mathrm{H}$ NMR spectrum of polyCP made of commercial CP shows minor olefinic proton signals around the major

12 signal of polyCP, which were undetected from the cyclic polyCP made of purified CP (Fig. 3e). The 13 integration of the minor signals was calculated to $1900 \mathrm{ppm}$, which is close to the amount of

14 linear olefin impurities in commercial CP stock, implying the undesired chain transfer by the 15 impurities. This negative effect on topology was also corroborated by DEPT $135{ }^{13} \mathrm{C}$ NMR spectroscopy, where ill-defined side signals were detected in the case of REMP of commercial CP

17 (Fig. S3). These results highlighted that the use of a purer monomer is critical in REMP, where small changes of monomer purity make a big difference of topological purity.

A cyclic polymer dispenser: "monomer in, polymer out". Despite the simplified separation, there

22 the chelating benzylidene structure, catalysts regenerated after release of cyclic polyCP can result

23 in vulnerable alkylidene structures ${ }^{39}$; in the presence of such catalysts, the post-synthetic 
1 filtration requires anhydrous degassed washing solvents under inert conditions for the catalyst

2 recovery. In addition, the required amount of solvent increases as the amount or molar-mass of

3 polymer increases. This ex situ filtration endangers the catalysts and ultimately depreciates the

4 practicality of the process.

Just as many synthetic chemists such as Dean, Stark, Schlenk and Soxhlet did in the past, we anticipated that a well-designed glassware would be a game changer, which streamlines the

7 process. Our initial attempt was to use a commercial solid-liquid extractor, known as the Soxhlet

8 extractor, where Het-D was placed with a cellulose thimble as a cartridge in the extraction

9 chamber, and then CP was refluxed in neat under an argon atmosphere. While CP was

10 polymerized as a monomer inside of the thimble, unreacted CP as a solvent washed the released

11 cyclic polyCP off the thimble, brought the filtered polymer to a collection flask, and was then

12 freshly distilled back to the thimble. Since all the active catalytic species always remained in the

13 thimble under the inert atmosphere, this in situ separation system provided the possible

14 maximum level of the catalyst security.

Nevertheless, two drawbacks were realized. One was that the reaction temperature was dictated by boiling points of refluxing monomer/solvent. This is a problem since in the REMP of

$17 \mathrm{CP}$, the ceiling temperature is low $\left([\mathrm{M}]_{\mathrm{eq}}=1.17\right.$ at $\left.30^{\circ} \mathrm{C}^{40}\right)$. Indeed, the REMP of neat $\mathrm{CP}(\mathrm{bp}=45$

$18{ }^{\circ} \mathrm{C}$ ) in a Soxhlet extractor afforded cyclic polyCP not exceeding the molar mass of $20 \mathrm{kDa}$. The 19 other drawback was that the collection flask always contained a mixture of cyclic polyCP and CP, 20 which required an additional step for CP recovery. 

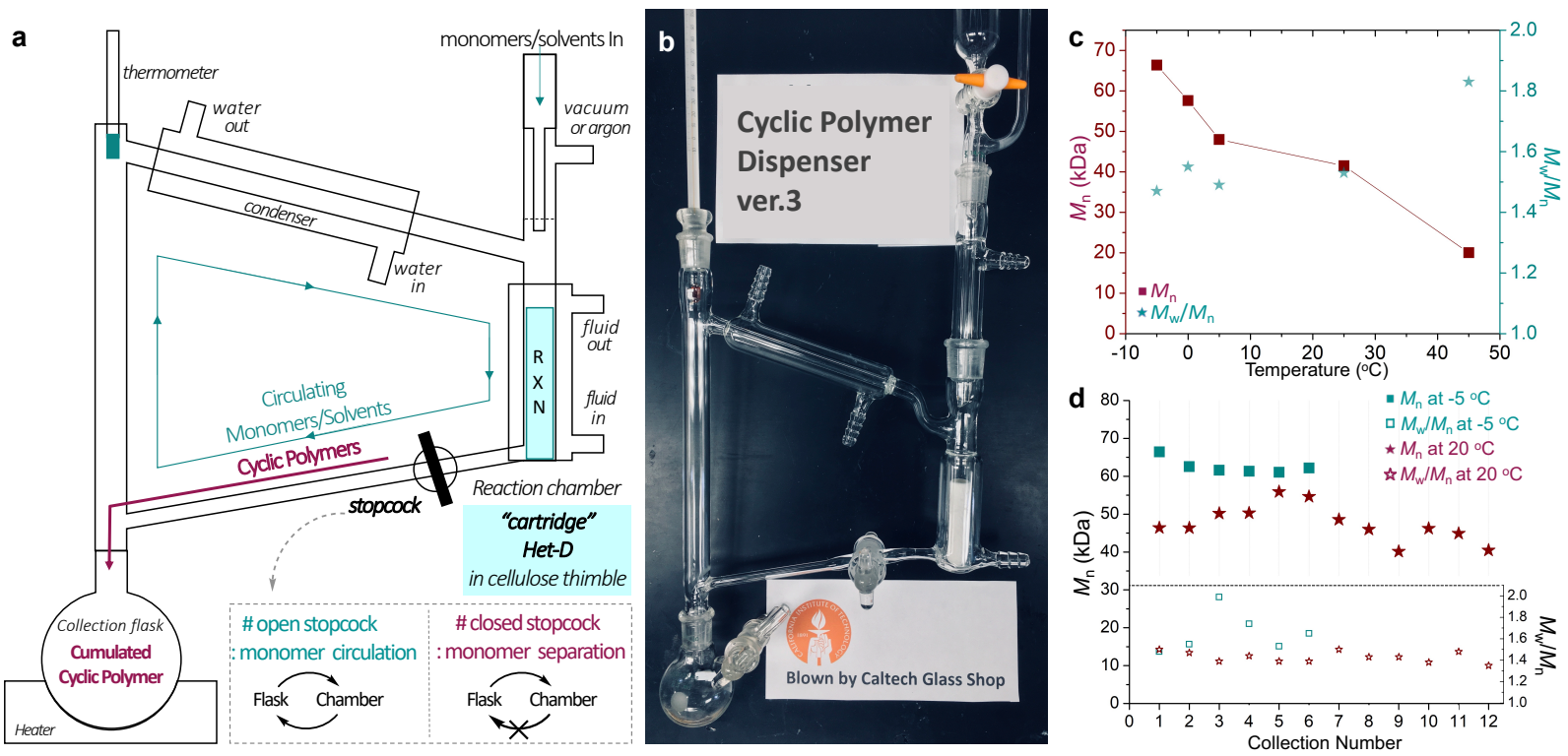

2 Fig. 4. "Monomer in, Polymer out". a, Schematic illustration of the cyclic polymer extractor mechanism. b, Photo of the extractor. c, Temperature dependence of REMP of CP d, Multiple collection of duplicate cyclic polyCPs via the dispenser.

Accordingly, an apparatus that is a hybrid of the distillation apparatus and the Soxhlet extractor was customized with two intended features (Fig. 4b, see Fig. S4 for our initial drawing and preliminary versions). The custom glassware, namely a cyclic polymer dispenser, consists of a fluid jacket surrounding the reaction chamber so that the reaction temperature can be controlled by a recirculating chiller/heater (Fig. 4a). By decreasing the reaction temperature, increased molar masses of cyclic polyCP up to $M_{\mathrm{n}}=66.4 \mathrm{kDa}$ were observed, showing that the

11 molar mass is thermodynamically controllable (Fig. 4c) ${ }^{33}$. Another feature is to segregate the 12 monomer and the polymer in the collection flask by closing a stopcock installed on the pathway 13 between the reaction chamber and the collection flask (Fig. 4a). When the stopcock is closed, CP 14 distilled from the flask into the reaction chamber cannot return to the flask, which leaves cyclic polyCP in the flask. Once the thermometer temperature drops (indicating the end of $\mathrm{CP}$ distillation), the flask filled with cyclic polyCP is rapidly switched to an empty flask under an 
1 increased argon pressure, and then the stopcock is reopened, which resumes CP circulation (and

2 additional $\mathrm{CP}$ is added to maintain monomer levels).

3 After mounting a cartridge on the cyclic polymer dispenser - i.e. a cellulose thimble

4 containing $100-\mathrm{mg}$ Het-D (400 nmol Ru) - we began to operate the process by adding CP. The

5 stopcock was closed whenever cyclic polyCP needed to be collected, and then reopened. After

6 collecting cyclic polyCPs 12 times that were dispensed to collection flasks, we obtained 11.3

7 grams of combined cyclic polyCP in overall $73 \%$ yield based on recovered CP (415,000 catalytic

8 turnovers). The amount of residual CP in the flasks, which was insufficient to be recovered by

9 distillation, caused the yield loss upon sample drying. Each collection offered a cyclic polyCP with

10 similar molar masses (Fig. 4d). High purity of cyclic topology of each round was confirmed with

11 SEC-viscometry and DEPT $135{ }^{13} \mathrm{C}$ NMR analysis (Fig. S5). The polymers were white with negligible

12 sign of metal residue $(<10 \mathrm{ppb})$ (Table S1).

13 It is noted that the catalyst was kept on duty, "quarantined" safely in the cellulose

14 cartridge under an argon atmosphere throughout the whole process. In many cases, regenerated

15 catalysts are too vulnerable to recycle in a conventional way which includes an ex situ step of off-

16 duty catalyst recovery. The use of the cyclic polymer dispenser showcases a new approach to

17 preserve the reusability of precious REMP catalysts by quarantining them in situ, as

18 demonstrated by constant activity over multiple collections with high total turnover number. In

19 addition, it can be extended for the synthesis of cyclic polymers with higher molar masses by

20 switching CP to higher ring-strain monomers such as cyclooctadiene (Fig. S6). 
"Social distancing" Effect of the immobilized catalyst. We came to realize a noncyclic catalyst in

2 which only the benzylidene moiety is tethered to the silica (Het-S in Fig. 2a) can be as effective

3 as Het-D, since only cyclic polymers can be released from the support by backbiting. If the

4 ruthenium loading on silica gel is sufficiently low, "socially distancing" ruthenium centers may

5 exclusively allow the desired backbiting pathway, physically disallowing the undesired interchain

6 interaction pathway that would release linear polymeric ruthenium complexes unless the silica

7 support holds both the NHC and benzylidene parts (Fig. 5a). On the other hand, if densely

8 populated, the propagating ruthenium could undergo this undesired pathway to release linear

9 defects because of chain proximity.

a

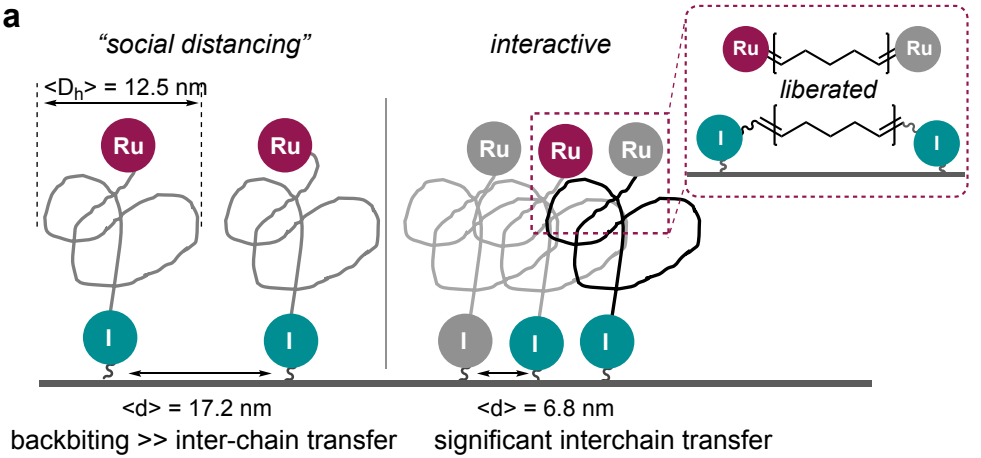

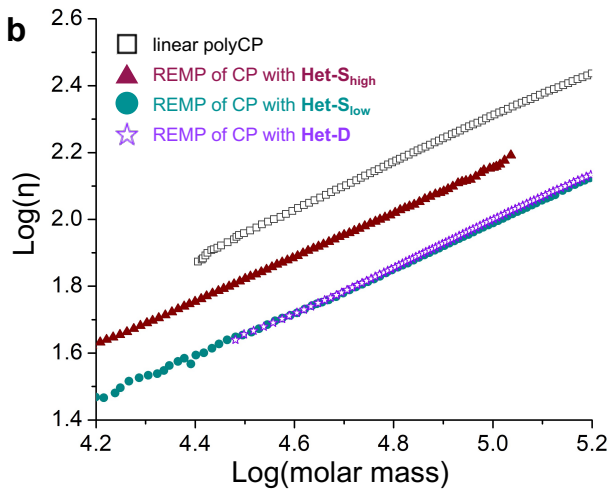

Fig. 5. "Social distancing" effect. a, Schematic illustration of the propensity for the interchain interaction affected by ruthenium density on silica gel. $\mathbf{b}$, Mark-Houwink-Sakurada plots of cyclic polyCP made with Het-D, Het-S low $_{\text {and }}$ Het-S high.

To demonstrate the "social distancing" effect, densely populated Het-S (Het-S high, 18 $\mu \mathrm{mol} \mathrm{Ru} / 1 \mathrm{~g}$ silica) and a low populated catalyst (Het-S low, $_{\text {. }} .8 \mu \mathrm{mol} \mathrm{Ru} / 1 \mathrm{~g}$ silica) were prepared. Based on surface area of silica gel, the average ruthenium distance of Het-S $S_{\text {low }}$ was estimated to be $17.2 \mathrm{~nm}$, which is larger than the hydrodynamic diameter $(12.5 \mathrm{~nm} \pm 0.6)$ of the isolated cyclic 
1 polyCP measured in $\mathrm{CP}$ at $22{ }^{\circ} \mathrm{C}$ by dynamic light scattering, whereas the ruthenium distance of

2 Het-S $S_{\text {high }}$ was $6.8 \mathrm{~nm}$, indicating more congested environment (Table S5).

All characterization data of two cyclic polyCPs obtained with Het-D and Het-Slow - molar masses, the Mark-Houwink-Sakurada plots, the conformation plots, ${ }^{1} \mathrm{H}$ NMR and DEPT $135{ }^{13} \mathrm{C}$

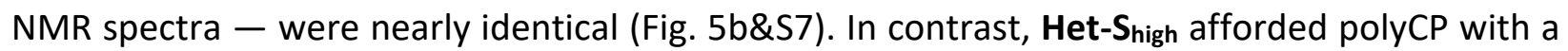
higher intrinsic viscosity than Het-Slow, which implied more interchain transfer events. DEPT 135

$7 \quad{ }^{13} \mathrm{C}$ NMR spectrum also manifested ill-defined side signals. ICP-MS detected 2000 times higher

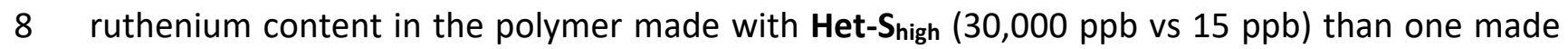

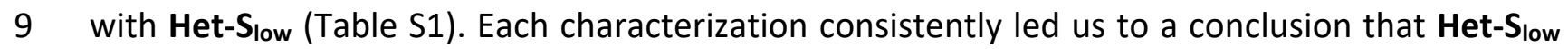
10 provided cyclic polyCP with as pure topology as does Het-D, while "REMP" of CP with Het-Shigh 11 suffered from the undesired side pathways. The discovery of the "social distancing" effect paves

12 the way for potential manipulation of catalytic activity/selectivity of the catalysts by modifying 13 the $\mathrm{NHC}^{41}$. In addition, noncyclic silica-supported catalysts are easier to prepare, which increases 14 the practicality of the system.

16 Hydrogenation of cyclic polyCP to cyclic polyethylene. The isolated cyclic polyCP was

17 hydrogenated to cyclic polyethylenes using the tosylhydrazine decomposition method ${ }^{11}$. The full 18 conversion was confirmed by solid-state ${ }^{13} \mathrm{C}$ NMR analysis (Fig. S8). The cyclic polyethylene had 19 a slightly lower melting point $\left(\mathrm{T}_{\mathrm{m}}=131^{\circ} \mathrm{C}\right)$ and crystallization point $\left(\mathrm{T}_{\mathrm{c}}=113^{\circ} \mathrm{C}\right)$ when compared 20 with its linear analog $\left(\mathrm{T}_{\mathrm{m}}=133^{\circ} \mathrm{C}, \mathrm{T}_{\mathrm{c}}=115^{\circ} \mathrm{C}\right)$ (Fig. S9), which is an opposite trend to our previous 21 observation with higher-molar mass cyclic polyethylenes $\left(T_{m}=132{ }^{\circ} \mathrm{C}, T_{c}=115^{\circ} \mathrm{C}\right.$ vs for the cyclic 
one vs. $\mathrm{T}_{\mathrm{m}}=130^{\circ} \mathrm{C}, \mathrm{T}_{\mathrm{c}}=113^{\circ} \mathrm{C}$ for the linear one $)^{11}$. This discrepancy as well as other properties of

2 cyclic polyethylene are currently under thorough investigation.

3

4

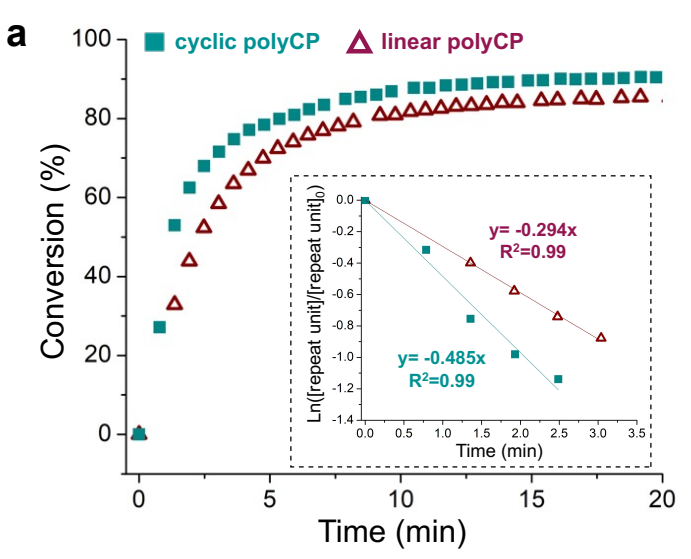

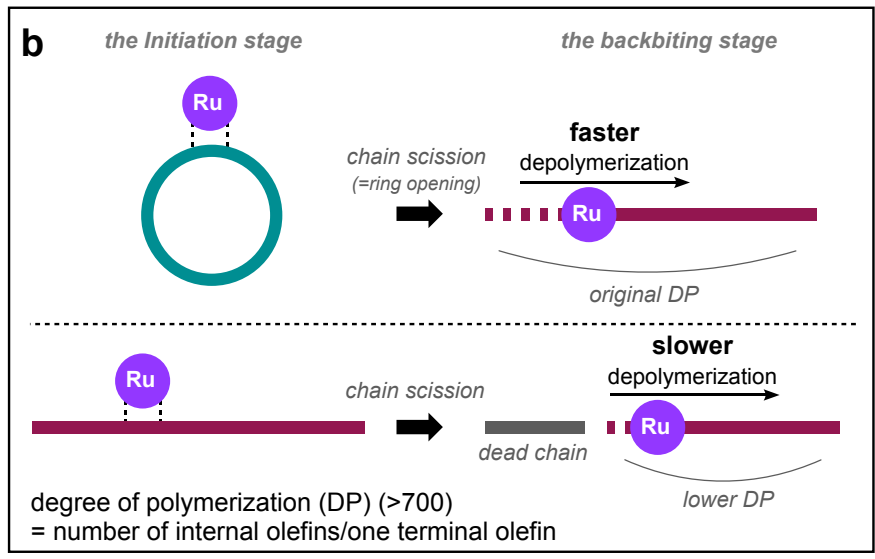

5 Fig. 6. Topology dependence of depolymerization. a, Depolymerization conversions of cyclic polyCP

$6\left(M_{\mathrm{n}}=56.8 \mathrm{kDa}, M_{\mathrm{w}} / M_{\mathrm{n}}=1.37\right)$ and linear polyCP $\left(M_{\mathrm{n}}=52.8 \mathrm{kDa}, M_{\mathrm{w}} / M_{\mathrm{n}}=1.66\right)$. conditions: ${ }^{1} \mathrm{H} \mathrm{NMR}, \mathrm{CDCl}_{3}$

7 (25 mM based on the repeat unit), 0.5 mol\% Grubbs $1^{\text {st }}$ generation catalyst, room temperature. b, A

8 proposed rationale of the expedited polymerization rate of cyclic polyCP.

Depolymerization kinetics: cyclic polyCP vs linear polyCP. One of the merits of polyCP is its

11 chemical recyclability to monomer in the presence of an olefin metathesis catalyst, which permits

12 a circular polymer economy ${ }^{42}$. When cyclic and linear polyCPs with similar molar masses were

13 treated respectively with the $1^{\text {st }}$ generation Grubbs catalyst in chloroform, the cyclic polyCP was

14 found to depolymerize 1.6 times faster than its linear analogue $\left(k_{\text {depolymerization }}=-0.485 \mathrm{~min}^{-1}\right.$ for

15 cyclic polyCP vs $-0.294 \mathrm{~min}^{-1}$ for linear polyCP in Fig. 6a\&S10). Considering that the initiation

16 process occurs dominantly by random chain cleavages of abundant internal olefins over terminal

17 olefins (degree of polymerization (DP) $>700$ ), we tentatively believe that after the first metathesis 
1 ruthenium carbene and the other without it (Fig. 6b) ${ }^{43,44}$. On the other hand, cyclic polyCP would

2 be always opened to active linear polyCP with the original DP without dead fragments ${ }^{10}$, and this

3 expedited depolymerization. Research on the detailed topology effect depending on other

4 variables (molar mass, temperature, solvent, etc.) is ongoing.

6 Conclusion

7 We have developed a convenient synthetic approach for the synthesis of cyclic 8 polycyclopentenamer by leveraging heterogeneity of the catalysts with the help of the newly9 designed glassware. Once the heterogeneous catalyst was placed in the thimble and 10 cyclopentene was added to the reaction apparatus, the circular loop of polymerization, polymer 11 separation, and catalyst recovery operated on its own, and then dispensed cyclic 12 polycyclopentenamer into the collection flask. This vending-machine-like process not only 13 minimized manual labor but also maximized security and reusability of vulnerable REMP catalysts,

14 purity of cyclic topology, as well as scalability of polymer. In addition, the first example that a 15 cyclic polymer depolymerizes faster than its linear analogue was discussed, with which we hope 16 to give an intriguing insight to promote the circular polymer economy. Currently, preliminary 17 tests of cyclic polymers as a lubricant material are ongoing in our laboratories.

References

201 González-Reyes, G. A., Bayo-Besteiro, S., Vich Llobet, J. \& Añel, J. A. Environmental and economic constraints on the use of lubricant oils for wind and hydropower generation: the case of NATURGY. Sustainability 12, 4242 (2020). 
12 Wakiru, J., Pintelon, L., Muchiri, P. N., Chemweno, P. K. \& Mburu, S. Towards an innovative

$2 \quad$ lubricant condition monitoring strategy for maintenance of ageing multi-unit systems.

3 Reliab. Eng. Syst. 204, 107200 (2020).

43 Zolper, T. et al. Lubrication properties of polyalphaolefin and polysiloxane lubricants:

5 molecular structure-tribology relationships. Tribol. Lett. 48, 355-365 (2012).

64 Greaves, M. Pressure viscosity coefficients and traction properties of synthetic lubricants

7 for wind turbine gear systems. Lubr. Sci. 24, 75-83 (2012).

85 Ray, S., Rao, P. V. C. \& Choudary, N. V. Poly- $\alpha$-olefin-based synthetic lubricants: a short 9 review on various synthetic routes. Lubr. Sci. 24, 23-44 (2012).

106 Martini, A., Ramasamy, U. S. \& Len, M. Review of viscosity modifier lubricant additives. 11 Tribol. Lett. 66, 58 (2018).

127 Morgan, S., Ye, Z., Subramanian, R. \& Zhu, S. Higher-molecular-weight hyperbranched polyethylenes containing crosslinking structures as lubricant viscosity-index improvers.

14 Polym. Eng. Sci. 50, 911-918 (2010).

158 Ver Strate, G. \& Struglinski, M. J. in Polymers as rheology modifiers Vol. 462 ACS Symposium Series Ch. 15, 256-272 (American Chemical Society, 1991).

179 Peterson, G. I. \& Choi, T.-L. The influence of polymer architecture in polymer mechanochemistry. Chem. Commun. 57, 6465-6474 (2021).

1910 Lin, Y., Zhang, Y., Wang, Z. \& Craig, S. L. Dynamic memory effects in the mechanochemistry 20 of cyclic polymers. J. Am. Chem. Soc. 141, 10943-10947 (2019).

2111 Bielawski, C. W., Benitez, D. \& Grubbs, R. H. An “endless" route to cyclic polymers. Science 22 297, 2041-2044 (2002). 
112 Xia, Y. et al. Ring-expansion metathesis polymerization: catalyst-dependent 2 polymerization profiles. J. Am. Chem. Soc. 131, 2670-2677 (2009).

313 Boydston, A. J., Xia, Y., Kornfield, J. A., Gorodetskaya, I. A. \& Grubbs, R. H. Cyclic 4 ruthenium-alkylidene catalysts for ring-expansion metathesis polymerization. J. Am. $5 \quad$ Chem. Soc. 130, 12775-12782 (2008).

614 Xia, Y., Boydston, A. J. \& Grubbs, R. H. Synthesis and direct imaging of ultrahigh molecular $7 \quad$ weight cyclic brush polymers. Angew. Chem. Int. Ed. 50, 5882-5885 (2011).

815 Boydston, A. J., Holcombe, T. W., Unruh, D. A., Fréchet, J. M. J. \& Grubbs, R. H. A direct 9 route to cyclic organic nanostructures via ring-expansion metathesis polymerization of a dendronized macromonomer. J. Am. Chem. Soc. 131, 5388-5389 (2009).

1116 Bielawski, C. W., Benitez, D. \& Grubbs, R. H. Synthesis of cyclic polybutadiene via ringcontaminants. J. Am. Chem. Soc. 125, 8424-8425 (2003).

1417 Wang, T.-W., Huang, P.-R., Chow, J. L., Kaminsky, W. \& Golder, M. R. A cyclic ruthenium polymerization. J. Am. Chem. Soc. 143, 7314-7319 (2021).

1718 Miao, Z. et al. Cyclic polyacetylene. Nat. Chem. 13, 792-799 (2021).

1819 McGraw, M. L., Clarke, R. W. \& Chen, E. Y. X. Synchronous control of chain 19 length/sequence/topology for precision synthesis of cyclic block copolymers from monomer mixtures. J. Am. Chem. Soc. 143, 3318-3322 (2021).

2120 Niu, W. et al. Polypropylene: now available without chain ends. Chem 5, 237-244 (2019). 
121 Roland, C. D., Li, H., Abboud, K. A., Wagener, K. B. \& Veige, A. S. Cyclic polymers from

$2 \quad$ alkynes. Nat. Chem. 8, 791-796 (2016).

322 Lidster, B. J. et al. Macrocyclic poly( $p$-phenylenevinylene)s by ring expansion metathesis 4 polymerisation and their characterisation by single-molecule spectroscopy. Chem. Sci. 9, $5 \quad$ 2934-2941 (2018).

623 Zhang, K., Lackey, M. A., Wu, Y. \& Tew, G. N. Universal cyclic polymer templates. J. Am. $7 \quad$ Chem. Soc. 133, 6906-6909 (2011).

824 Edwards, J. P., Wolf, W. J. \& Grubbs, R. H. The synthesis of cyclic polymers by olefin 9 metathesis: achievements and challenges. J. Polym. Sci. A Polym. Chem. 57, 228-242 10 (2018).

1125 Chang, Y. A. \& Waymouth, R. M. Recent progress on the synthesis of cyclic polymers via ring-expansion strategies. J. Polym. Sci. A Polym. Chem. 55, 2892-2902 (2017).

1326 Haque, F. M. \& Grayson, S. M. The synthesis, properties and potential applications of $14 \quad$ cyclic polymers. Nat. Chem. 12, 433-444 (2020).

1527 Tuba, R. Synthesis of cyclopolyolefins via ruthenium catalyzed ring-expansion metathesis polymerization. Pure Appl. Chem. 86, 1685-1693 (2014).

1728 Jawiczuk, M., Marczyk, A. \& Trzaskowski, B. Decomposition of ruthenium olefin metathesis catalyst. Catalysts 10, 887 (2020).

1929 Allen, D. P., Van Wingerden, M. M. \& Grubbs, R. H. Well-defined silica-supported olefin 20 metathesis catalysts. Org. Lett. 11, 1261-1264 (2009).

2130 Dewaele, A., Van Berlo, B., Dijkmans, J., Jacobs, P. A. \& Sels, B. F. Immobilized Grubbs 22 catalysts on mesoporous silica materials: insight into support characteristics and their 
1 impact on catalytic activity and product selectivity. Catal. Sci. Technol. 6, 2580-2597

2

331 Hejl, A., Scherman, O. A. \& Grubbs, R. H. Ring-opening metathesis polymerization of 4 functionalized low-strain monomers with ruthenium-based catalysts. Macromolecules 38, 5 (2016). 7214-7218 (2005).

32 Neary, W. J. \& Kennemur, J. G. Polypentenamer renaissance: challenges and opportunities. ACS Macro Lett. 8, 46-56 (2019).

33 Tuba, R. \& Grubbs, R. H. Ruthenium catalyzed equilibrium ring-opening metathesis polymerization of cyclopentene. Polym. Chem. 4, 3959-3962 (2013).

34 Neary, W. J. \& Kennemur, J. G. Variable temperature ROMP: leveraging low ring strain thermodynamics to achieve well-defined polypentenamers. Macromolecules 50, 49354941 (2017).

35 Mulhearn, W. D. \& Register, R. A. Synthesis of narrow-distribution, high-molecular-weight ROMP polycyclopentene via suppression of acyclic metathesis side reactions. ACS Macro Lett. 6, 112-116 (2017).

36 Lee, L.-B. W. \& Register, R. A. Acyclic metathesis during ring-opening metathesis polymerization of cyclopentene. Polymer 45, 6479-6485 (2004).

37 Ji, S., Hoye, T. R. \& Macosko, C. W. Controlled synthesis of high molecular weight telechelic polybutadienes by ring-opening metathesis polymerization. Macromolecules 37, 5485-5489 (2004). 
138 Obligacion, J. V. \& Chirik, P. J. Bis(imino)pyridine cobalt-catalyzed alkene isomerization-

2

3

4

5 hydroboration: a strategy for remote hydrofunctionalization with terminal selectivity. J. Am. Chem. Soc. 135, 19107-19110 (2013).

39 Ulman, M. \& Grubbs, R. H. Ruthenium carbene-based olefin metathesis initiators: catalyst decomposition and longevity. J. Org. Chem. 64, 7202-7207 (1999).

40 Torre lii, M., Mulhearn, W. D. \& Register, R. A. Ring-opening metathesis copolymerization of cyclopentene above and below its equilibrium monomer concentration. Macromol. Chem. Phys. 219, 1800030 (2018).

41 Szczepaniak, G., Kosiński, K. \& Grela, K. Towards "cleaner" olefin metathesis: tailoring the NHC ligand of second generation ruthenium catalysts to afford auxiliary traits. Green Chem. 16, 4474-4492 (2014).

42 Coates, G. W. \& Getzler, Y. D. Y. L. Chemical recycling to monomer for an ideal, circular polymer economy. Nat. Rev. Mater. 5, 501-516 (2020).

43 Neary, W. J., Isais, T. A. \& Kennemur, J. G. Depolymerization of bottlebrush polypentenamers and their macromolecular metamorphosis. J. Am. Chem. Soc. 141, 14220-14229 (2019).

44 Yuan, J., Giardino, G. J. \& Niu, J. Metathesis cascade-triggered depolymerization of enyne self-immolative polymers. Angew. Chem. Int. Ed., doi:https://doi.org/10.1002/anie.202108239 (2021). 
Methods

2 Experimental details are provided in the Supplementary Information.

4 Procedure for the synthesis of cyclic polyCP. All glasses were dried in a $165^{\circ} \mathrm{C}$ oven prior to use.

5 The cyclic polymer dispenser was connected to a $25-\mathrm{mL}$ Schlenk flask, a thermometer, and a

6 dropping funnel with the help of suitable joints. The upper cooling jacket was equipped with a

7 water circulator, and the lower jacket was equipped with a circulating chiller or heater,

8 depending on the required reaction temperature. The detailed glassware setup is described with

9 photos in the Supplementary Information. The heterogeneous catalyst (Het-D or Het-S) was

10 weighed in air and placed in a commercial cellulose thimble. The thimble containing the catalyst

11 was transferred in the reaction chamber of the cyclic polymer dispenser. The whole glass system

12 was charged with argon by repetitive vacuum and argon backfilling. Afterwards, the purified CP

13 was added to the reaction chamber via the dropping funnel. The distillation column part was

14 wrapped with cotton and foils for heat insulation. The heater was set to $70^{\circ} \mathrm{C}$. For convenience,

15 a heating block was used instead of an oil bath. Once the circulation of CP began, the

16 thermometer turned to $45^{\circ} \mathrm{C}$. After 3-6 hours when the polymer needed to be collected, the

17 stopcock of the dispenser was closed. When the thermometer temperature dropped to room

18 temperature, the argon pressure was increased, and then the filled $25-\mathrm{mL}$ flask was replaced with

19 a new 25-mL Schlenk flask. On the switching process, the air contaminant was vented out of the

20 system by briefly opening the valve of the Schlenk flask. The argon pressure was reduced back to

21 normal, and the stopcock was reopened, and then the CP circulation resumed. The stopcock was

22 closed for the next collection and the procedure was reiterated. 


\section{Data availability}

All data supporting the findings of this study are available within the Article and its Supplementary Information.

4

\section{Acknowledgements}

The authors would like to thank all scientific communities for their cooperative fighting

7 against Covid-19. This work is financially supported by the National Science Foundation

8 (CHE\#1807154) and the Creative Research Initiative Grant and the Nano-Material Technology

9 Program through NRF (South Korea). Mr. Nathan Hart at Caltech Glass Shop is gratefully

10 acknowledged for the glass blowing. Dr. Sonjong Hwang is appreciated for the solid-state NMR.

11 Prof. Yan Xu (Peking University), Dr. Jung-Ah Song (Samsung), Ms. Yoon-Jung Jang (Univ. of

12 Minnesota), and Dr. Daryl Allen (Materia) are acknowledged for the helpful discussion and 13 encouragement.

\section{Author contributions}

R.H.G. and K.-Y.Y. conceived and designed the project. R.H.G. and T.-L.C. directed the

17 project and provided valuable input. K.-Y.Y, Q.G. and J.P.E synthesized the catalysts. K.-Y.Y

18 designed the glassware. K.-Y.Y., J.N. and Q.G. characterized the polymers. J.N. performed

19 depolymerization experiments. R.T. demonstrated the first heterogeneous cyclic polymer

20 process. All authors analyzed the data and discussed the results. K.-Y.Y prepared the initial

21 manuscript and then all authors reviewed and commented on the manuscript. K.-Y.Y., J.N. and

22 Q.G. contributed the project equally. 
2 Competing interests

3 The authors declare no competing interests. 